Math. Model. Nat. Phenom.

Vol. xx, No. xx, 2017, pp. 1-19

DOI: $\mathrm{xx} \cdot \mathrm{xxxx} / \mathrm{mmnp} / \mathrm{xxxxxxxx}$

\title{
Analysis of an Interneuron Gamma Mechanism for Cross-Frequency Coupling
}

\author{
M. Chehelcheraghi ${ }^{1 *}$, C. Nakatani ${ }^{1}$, C. van Leeuwen ${ }^{1,2}$ \\ ${ }^{1}$ Brain and Cognition Research Unit, KU Leuven, Leuven, Belgium \\ ${ }^{2}$ Center for Cognitive Science, TU Kaiserslautern, Kaiserslautern, Germany
}

\begin{abstract}
Signals with multiple oscillatory components may exhibit cross frequency coupling (CFC): a slow component modulating the amplitude and/or frequency of a fast one. CFC is ubiquitous in oscillatory brain signals but how it arises has remained unclear. We recently proposed a systematic approach to simulate $\mathrm{CFC}$, in which all common modulations are generated by a Neural Mass Model [7]. A key mechanism in this approach is the dynamic self-feedback circuit of the fast inhibitory interneuron population that generates Gamma band oscillatory activity. Depending on noise-input level, the circuit switches between a limit cycle regime enabling amplitude modulation and a resonance regime enabling frequency modulation. In this study, we analyze the behavior of this circuit, using the describing function method for the limit cycle regime and root locus analysis for the resonance regime. The relationship between circuit parameters and behavior is investigated by bifurcation analysis.
\end{abstract}

Keywords and phrases: Inhibitory Interneuron, dynamic self-feedback, Interneuron Gamma (ING), Describing Function, Root Locus

Mathematics Subject Classification: $92 \mathrm{~B} 25$

\section{Introduction}

Between different frequency components of oscillatory brain activity, cross-frequency coupling (CFC) is observed [6][14][20][23][24][27]. The coupling may occur between instantaneous phase, instantaneous amplitude and instantaneous frequency of two or more distinct oscillatory components of the same signal, or of two or more different signals. Several of these couplings are relevant to understand the relationship between brain activity and behavior [6][20][27]; Jensen and Colgin [23] highlight the following types of CFC as of interest: phase-phase, phase-frequency, phase-amplitude, amplitude-amplitude, frequencyfrequency, and amplitude-frequency couplings (PPC, PFC, PAC, AAC, FFC and AFC, respectively). However, their underlying mechanisms are still not fully understood. An approach which has been successful in modeling various oscillatory phenomena involves Neural Mass Models [11][12][22]. Neural Mass Models (NMMs) simulate key properties of electrical activity from mesoscopic neuronal populations, ranging from several hundreds to tens of millions of neurons, as recorded in electroencephalography (EEG) [22][28], Magnetoencephalography (MEG) [1][18] or local field potentials (LFP) [3][21][30]. In these models, population activity is represented by a small number of non-linear differential equations; oscillations result from interactions between several distinct types of neural populations, such as pyramidal neurons and inhibitory interneurons. NMMs have initially been used to simulate slow (Alpha and Theta frequency band) activity [22][28] and have subsequently been extended to higher frequency bands, such as Beta and Gamma [8][11][33][36][38]. As a prerequisite to CFC, an NMM should be able to generate at least

* Corresponding author. E-mail: mojtaba. chehelcheraghi@kuleuven. be 
two frequency components simultaneously: a slow and a fast one. Slow band activity is a basic property of these models that has been extensively discussed elsewhere [15][29][31]. However, the extension of these models to co-occurring fast activity is still not broadly established. We will discuss our proposal for how to include the fast component. Generally speaking, two mechanisms could be distinguished for generating these fast oscillations [5]: first, interaction of excitatory and inhibitory activity; the resulting activity is called pyramidal-interneuron Gamma (PING; [4][10][17][25]); second, self-inhibition within a population of inhibitory neurons. This results in, what is known as interneuron Gamma (ING; [4][35][37]). Recently, we proposed a NMM which implements the ING mechanism in a fast inhibitory interneuron population with dynamic self-feedback [8]. The proposed ING circuit has a key role in modeling five out of six forms of CFC [7] considered of interest [23]. Whether frequency or amplitude modulation occurs depends on the type of oscillation of the ING circuit. Depending on input level, the circuit was either inactive or showed forced or limit cycle oscillations. The forced oscillations provided a resonance regime, allowing the model to exhibit frequency modulation; the limit cycle oscillations allowed amplitude modulation. Our current aim is to analyze the behavior of the circuit; in particular its ability to bifurcate between forced and limit cycle oscillations. The results of the analysis certify our choice of parameter values in the proposed model [7] for obtaining frequency and amplitude modulation.

\subsection{Classification of Cross Frequency Coupling Forms}

The way in which the fast oscillation circuit leads to PFC, PAC, AAC, FFC and AFC depends on the configuration of the model in Chehelcheraghi et al [7]. The complexity of the NMM model needed for simulation of each of these types leads to the following classification. (I) The Zero Modulation Class consists of Phase-Phase coupling. In this class no modulation, neither in frequency nor amplitude, takes place. Two signals are phase-phase coupled if they are m:n synchronized (illustrated by the relation between $M_{1}$ and $M_{2}$ in Figure 1). PPC is only meaningful, when it occurs between simple monofrequency (narrow band) signals. (II) The One Modulation Class consists of Phase-Amplitude and Phase Frequency Coupling. In this class, the first of two signals is the fast signal. It is modulated in its frequency or amplitude by a slow signal. In Figure 1, Phase-Amplitude Coupling is illustrated by the relations in $M_{1}-X_{1}$ and $M_{1}-X_{2}$ and Phase-Frequency Coupling is shown by the relations in $M_{1}-Y_{1}$ and $M_{1}-Y_{2}$. (III) The Two Modulations Class consists of Amplitude-Amplitude, Frequency-Frequency and Amplitude-Frequency Couplings. In this class, two fast signals are modulated in their frequency or amplitude. The modulation (slow signal) on the frequency or amplitude of the first signal (fast signal) is synchronized with the modulations (slow signal) on the frequency or amplitude of the second signal (fast signal). While three signals (two fast and one slow) are involved in this class, the CFC is reported conventionally as a property of the two fast signals. In Figure 1, the Amplitude-Amplitude Coupling is illustrated in the relation $X_{1}-X_{2}$, Frequency-Frequency Coupling in the relation $Y_{1}-Y_{2}$ and AmplitudeFrequency Coupling in the relations $X_{1}-Y_{1}, X_{1}-Y_{2}, X_{2}-Y_{1}$ and $X_{2}-Y_{2}$ ).

\subsection{A Framework for Simulating Cross Frequency Coupling}

In the model of Chehelcheraghi et al [7], which is conceptualized in Figure 2, the mechanism for CFC generation consists of three component functions: Generation of Oscillations, Synchronization, and Modulation (on amplitude and \or frequency). Whether Synchronization and Modulation need to be invoked, however, depends on the type of CFC. E.g. the first class of CFC only incorporates Generation and Synchronization, and does not involve Modulation (Figure 2a). For simulating the second class of CFC, two oscillatory signal components are generated, of which the slower one modulates the amplitude or frequency of the faster one. These modulations can both be generated within a single region. When it comes to cross regional modulation, i.e. the sources of slow and fast oscillations are spatially far, Synchronization is needed as well (Figure 2b). The third class of CFC is necessarily a cross regional phenomenon (Figure 2c). Thus, it requires all the three component mechanisms.

The first class leads in the model to PPC between the slow signals. Since it lacks any fast oscillations and correspondingly no modulation, this class will not further be considered here. The second and third class are realized in the model by switching between different modes of the ING circuit. Since the fast oscillations in CFC are either turned-off or are modulated in their frequency or amplitude, the ING circuit should be able to operate in three modes: 1 . Inactive 2 . frequency modulation, and 3 . amplitude modulation. The frequency and amplitude modulation modes are obtained with, respectively, forced 


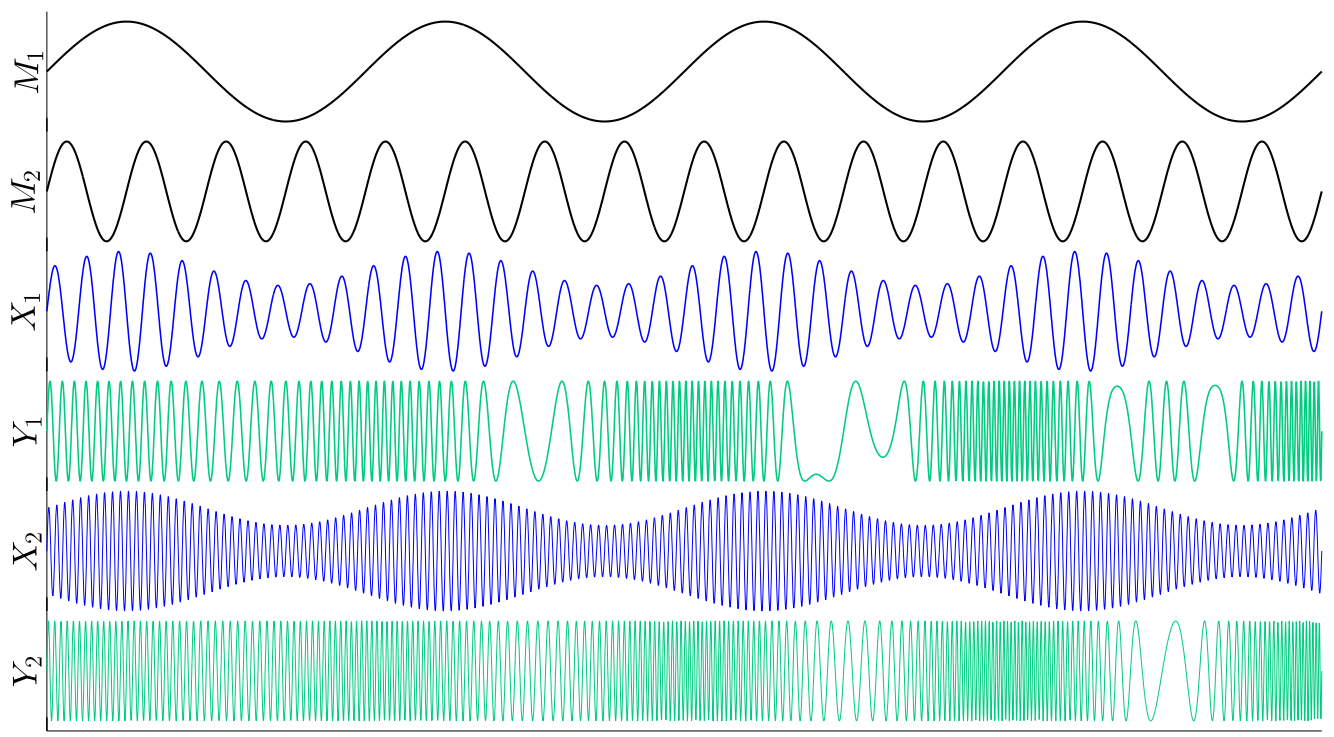

FiguRE 1. Different forms of cross-frequency coupling. $M_{1}$ and $M_{2}$ are non-modulated signals which are 1:3 synchronized, meaning that in each cycle of $M_{1}$ three cycles of $M_{2}$ occur. $X_{1}$ and $X_{2}$ are representing two amplitude modulated signals in which the amplitude modulations are coordinated in time with $M_{1} . Y_{1}$ and $Y_{2}$ are signals frequency modulated by M1.

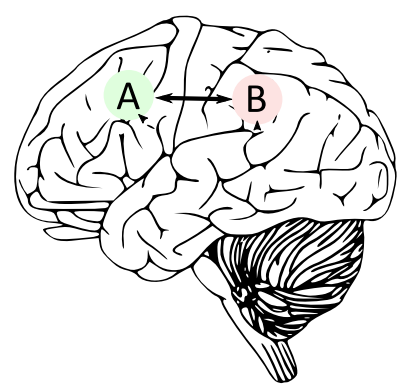

(a) Zero Modulation Class
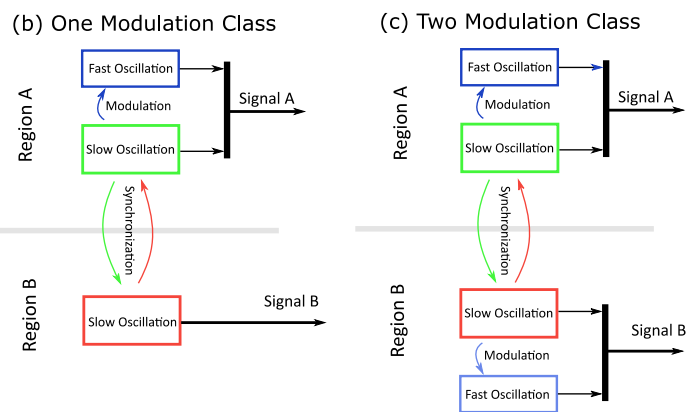

Figure 2. Conceptualization of Different Classes of Cross-Frequency Coupling (CFC) in mesoscopic brain recordings (e.g., EEG, MEG or LFP). CFC between two spatially distinct recorded signals, i.e. region A and Region B, can be categorized into three classes: (a) Zero modulation class: the sources of fast oscillations are inactive in both regions and the slower oscillations of the regions are m:n synchronized (b) One modulation class: The source of fast oscillation is active in only one region, i.e. region A. In local CFC the slower oscillation modulates the amplitude and/or frequency of the faster oscillation of the same region. Cross regional CFC happens when the slower oscillations of both regions get synchronized. (c) Two modulation class: The sources of fast oscillations are active in both regions. The slower oscillation in each region modulates the amplitude and/or frequency of the same region. The CFC occurs between the fast oscillations, when the slower modulatory oscillations synchronize across the regions.

and limit cycle oscillations. The first occurs when all the state variables of the non-linear system are exponentially converging. Consequently, the state variables of the system will dwell around their fixed points. Therefore the transfer function of the nonlinear system can be calculated around these fixed points using linearization. When excited, the system will respond in its resonance frequency. We will analyze this behavior using the root locus analysis method and show that this behavior is suitable for frequency modulation. The second type of oscillation occurs if there is a closed orbit in the phase space of the system state variables. In this case some state variables of the system are not converging to a fixed 
value, but their values change periodically and form a stable oscillation. The limit-cycle oscillation can be analyzed by using the method of describing functions. We will show that this behavior is suitable for simulating amplitude modulation. The switch between the two modes will be studied using bifurcation analysis methods.

\section{Materials and Methods}

\subsection{ING Circuit}

The ING circuit is composed of three basic blocks: sigmoid function, dendritic transfer function and dynamic self-feedback. The block diagram of the ING circuit is given in Figure 3a. The sigmoid function converts the inhibitory interneuron mean membrane potential, $v_{m}(t)$, to expected firing rate. The sigmoid function is described by:

$$
\operatorname{Sig}\left(v_{m}(t)\right)=\frac{\nu_{\max }}{1+e^{-r\left(v_{m}(t)-v_{t h}\right)}}
$$

In which the parameter $\nu_{\max }$ is the maximum firing rate of the population of neurons, $v_{t h}$ is the value of the potential for which a 50 percent mean firing rate is achieved, and $\mathrm{r}$ is the slope of the sigmoid at $v=v_{t h} ; v_{t h}$ represents the mean firing threshold. An external input, $P_{m}^{*}(t)$, modulates the mean firing rate of the inhibitory interneuron. $P_{m}^{*}(t)$ models the modulations on the firing rate which are voltageindependent, e.g. ligand-gating [19][26] and/or temperature-gating [13][34]. The parameter $P_{m}^{*}(t)$ takes a negative sign as it represents the excitatory influence on the inhibitory population; Therefore, the net mean firing rate of the population is obtained according to following equation:

$$
f r(t)=\operatorname{Sig}\left(v_{m}(t)\right)-P_{m}^{*}(t)
$$

The net mean firing rate is converted to the mean post synaptic potential $\left(v_{1}\right)$ through the dendritic transfer function. The differential equation for the dendritic transfer function is:

$$
\frac{d^{2} v_{1}}{d t^{2}}=G_{u} \omega_{u} f r(t)-2 \omega_{u} \frac{d v_{1}}{d t}-\omega_{u}^{2} v_{1}
$$

in which $G_{u}$ and $\omega_{u}$ characterize the mean strength and speed, respectively, of the synaptic transfer function. This second-order differential equation can be rewritten as a system of two first-order equations:

$$
\begin{gathered}
\frac{d i}{d t}=G_{u} \omega_{u} f r(t)-2 \omega_{u} i-\omega_{u}^{2} v_{1} \\
\frac{d v_{1}}{d t}=i
\end{gathered}
$$

A self-feedback dynamic mechanism with plasticity time constant $\tau_{u}$, acts on the fast inhibitory population. The self-feedback block converts the mean post-synaptic potential $\left(v_{1}\right)$ to the unweighted selfinhibition potential $\left(v_{2}\right)$. The feedback uses a first order dynamic and it is described by:

$$
\frac{d v_{2}}{d t}=\frac{-1}{\tau_{u}} v_{2}+\frac{1}{\tau_{u}} v_{1}
$$

The dynamics in the feedback accounts for the plasticity of the self-synaptic gain. $v_{2}$ is weighted by $C_{f b}$, the strength of the self-feedback, and gives the self-inhibition potential, i.e. $C_{f b} v_{2}$. A second external input, $I_{u}(t)$, which presents the external pre-synaptic potentials excites the system as well; $I_{u}(t)$ together with the self-inhibition potential forms the mean membrane potential of the ING circuit, i.e. $v_{m}(t)=C_{f b} v_{2}+I_{u}(t)$. Note that for $C_{f b}=0$ or $\tau_{u} \rightarrow \infty$ the population has no feedback and the structure is reduced to the classical neural population model [11][22][28][36]. For $\tau_{u}=0$ the feedback is constant and the synaptic plasticity is ignored. For further analysis, it is convenient to merge the two external variable inputs $\left(P_{m}^{*}(t)\right.$ and $\left.I_{u}(t)\right)$ into a single variable, which represents the net external influence on the firing rate. The new variable involves the non-voltage gating part, $P_{m}^{*}(t)$, and voltagedependent gating mechanism, i.e. the influence of $I_{u}(t)$ on the firing rate. We denote the corresponding influence of $I_{u}(t)$ on the firing rate with the variable $P_{m}^{* *}(t)$. Once the behavior of the circuit is calculated for any given inputs $I_{u}(t)$ and $P_{m}^{*}(t)$, the variable $P_{m}^{* *}(t)$, can be calculated according to $P_{m}^{* *}(t)=$ 
$\operatorname{Sig}\left(C_{f b} v_{2}(t)\right)-\operatorname{Sig}\left(C_{f b} v_{2}(t)+I_{u}(t)\right)$. Therefore, without loss of generality, for the purpose of analysis we can re-write the equations of the ING circuit with a single input, $P_{m}(t)$, as follows:

$$
\begin{gathered}
\frac{d i}{d t}=G_{u} \omega_{u} \operatorname{Sig}\left(C_{f b} v_{2}\right)-G_{u} \omega_{u} P_{m}(t)-2 \omega_{u} i-\omega_{u}^{2} v_{1} \\
\frac{d v_{1}}{d t}=i \\
\frac{d v_{2}}{d t}=\frac{-1}{\tau_{u}} v_{2}+\frac{1}{\tau_{u}} v_{1}
\end{gathered}
$$

in which $P_{m}(t)=P_{m}^{*}(t)+P_{m}^{* *}(t)$. We consider $P_{m}(t)$ as the main input of the ING circuit. $P_{m}(t)$ can be decomposed into a constant $\left(P_{u}\right)$ and a time-variant $\left(p_{u}(t)\right)$ part: $P_{m}(t)=P_{u}+p_{u}(t)$. The schematic representation of the Equations (2.7-2.9) is shown in Figure 3b.
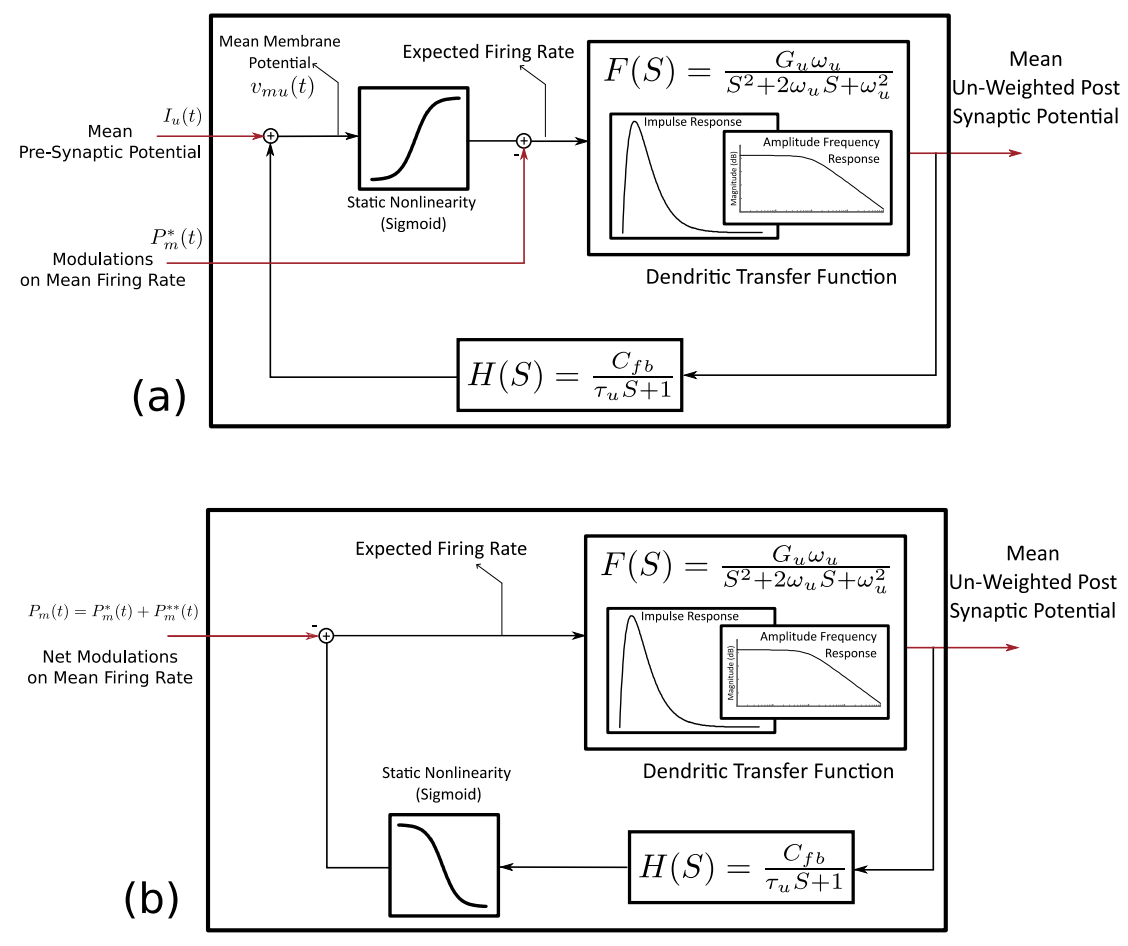

FIGURE 3. Schematic of the ING circuit. (a) block diagram representation of Equations 4-6 (b) block diagram representation of Equations 7-9.

\subsection{Dimension Reduction}

Without loss of generality, we change the variables as follows:

$$
\begin{gathered}
t^{\prime}=\omega_{u} t \\
v_{2}^{\prime}=r C_{f b} v_{2} \\
i^{\prime}=\frac{1}{G_{u} \omega_{u}} i \\
v_{1}^{\prime}=\frac{1}{G_{u}} v_{1}
\end{gathered}
$$


Therefore, we obtain the dimensionless equations:

$$
\begin{gathered}
\frac{d i^{\prime}}{d t^{\prime}}=\frac{\nu_{\max } / \omega_{u}}{1+e^{r v_{t h}} e^{-v_{2}^{\prime}}}-\frac{P_{m}\left(t^{\prime}\right)}{\omega_{u}}-2 i^{\prime}-v_{1}^{\prime} \\
\frac{d v_{1}^{\prime}}{d t^{\prime}}=i^{\prime} \\
\frac{d v_{2}^{\prime}}{d t^{\prime}}=\frac{-1}{\tau_{u} \omega_{u}} v_{2}^{\prime}+\frac{r C_{f b} G_{u}}{\tau_{u} \omega_{u}} v_{1}^{\prime}
\end{gathered}
$$

We reparametrize the equations as follows:

$$
\begin{gathered}
\frac{d i^{\prime}}{d t^{\prime}}=\frac{\Gamma}{1+K_{0} e^{-v_{2}^{\prime}}}-P\left(t^{\prime}\right)-2 i^{\prime}-v_{1}^{\prime} \\
\frac{d v_{1}^{\prime}}{d t}=i^{\prime} \\
\frac{d v_{2}^{\prime}}{d t}=-\psi v_{2}^{\prime}+\psi C v_{1}^{\prime}
\end{gathered}
$$

In which:

$$
\begin{gathered}
\Gamma=\nu_{\max } / \omega_{u}=2 e_{0} / \omega_{u} \\
\psi=\frac{1}{\tau_{u} \omega_{u}} \\
C=r C_{f b} G_{u} \\
P\left(t^{\prime}\right)=\frac{P_{m}\left(t^{\prime}\right)}{\omega_{u}} \\
K_{0}=e^{r v_{t h}}
\end{gathered}
$$

\subsection{Fixed Points}

The fixed points can be obtained with the assumption that the time-variant part of $P_{m}(t)$ is zero. Therefore $P=\frac{P_{u}}{\omega_{u}}$ and we have:

$$
\begin{gathered}
i=0 \\
\psi \neq 0, v_{2}^{\prime}=C v_{1}^{\prime} \\
v_{1}^{\prime}=\frac{\Gamma}{1+K_{0} e^{-v_{2}^{\prime}}}-P
\end{gathered}
$$

Therefore, any singular point $\mathrm{S}$ can be written as a function of $v_{2}^{\prime}$ :

$$
S\left(v_{2}^{\prime}\right)=\left(0, \frac{v_{2}^{\prime}\left(\Gamma, K_{0}, C, P\right)}{C}, v_{2}^{\prime}\left(\Gamma, K_{0}, C, P\right)\right)
$$

where $v_{2}^{\prime}$ is a function of $\Gamma, K_{0}, C$ and $P$. Although singular points cannot be written explicitly as function of all model parameters, the model parameters and $v_{2}^{\prime}$ are related according to:

$$
P=\frac{\Gamma}{1+K_{0} e^{-v_{2}^{\prime}}}-\frac{v_{2}^{\prime}}{C}
$$


Note that the singular points are independent of the value of $\psi$. The Jacobian matrix of the linearized system near its fixed points is:

$$
J=\left[\begin{array}{ccc}
-2 & -1 & \Pi \\
1 & 0 & 0 \\
0 & \psi & -\psi
\end{array}\right]
$$

for which:

$$
\Pi\left(v_{2}^{\prime}\right)=\Pi\left(\Gamma, K_{0}, C, P\right)=\frac{K_{0} \Gamma e^{-v_{2}^{\prime}}}{\left(1+K_{0} e^{-v_{2}^{\prime}}\right)^{2}}
$$

\subsection{Parameter Range, Stability and Bifurcation Analysis}

The eigenvalues of the matrix in (2.30) are obtained by solving the characteristic equation:

$$
\lambda^{3}+(2+\psi) \lambda^{2}+(2 \psi+1) \lambda+\rho \psi=\lambda^{3}+a \lambda^{2}+b \lambda+c=0
$$

where $\rho=(1-C \Pi)$ is always positive and we have $\Pi_{\max }=\frac{\Gamma}{4}$ according to Equation $(2.31)$. The upper limit for the values of the parameter $|C \Pi|=\left|\frac{r \nu_{\max } G C_{f}}{4 \omega}\right|$ is restricted to keep the model parameters within the ratios proposed in previous studies [9][18]. Accordingly, we assumed an upper limit of $\Lambda=20$. While the parameters $r, G, \omega_{u}$ and $\nu_{\max }$ are positive, the parameter $C_{f b}$ may, in principle, take negative or positive signs, for representing self-inhibition or self-excitation respectively. Whereas self-inhibition would preserve the intended interpretation of the circuit as inhibitory, self-excitation would render the circuit excitatory. We will show that the latter does not provide oscillations, and hence no model solutions are available if the circuit is excitatory. The parameter-range of $\Psi>1$ is not of interest; for this range the cut-off frequency of the frequency dependent synaptic depression is higher than that of the membrane potential. Therefore, modeling of synaptic depression (first-order dynamic in the feedback) will not be feasible. Thus, the solutions for the characteristic equation (Equation 2.32) are studied within biologically meaningful ranges of coefficient parameters, i.e., $-\Lambda+1<\rho<\Lambda+1$ and $0<\psi<1$. For $-\Lambda+1<\rho<1$ the self-feedback is excitatory $\left(C_{f b}>0\right)$, for $1<\rho<\Lambda+1$ the self-feedback is inhibitory $\left(C_{f b}<0\right)$, and for $\rho=1$ there is no self-feedback $\left(C_{f b}=0\right)$.

Applying the Routh-Horvitz criteria, for the condition in which $0<\rho<\frac{(2+\psi)(2 \psi+1)}{\psi}$ the system is exponentially convergent. The characteristic equation of the Jacobian matrix can be re-written as follows:

$$
(\lambda+1)^{2}(\lambda+\psi)=\psi(1-\rho)
$$

A graphical representation of the equation is shown in Figure 4 , where the curve equals $y_{1}=(\lambda+$ $1)^{2}(\lambda+\psi)$ and $y_{2}=\psi(1-\rho)$ is a horizontal line. We consider the following cases for the eigenvalues.

2.4.1. Excitatory Self-Feedback $(-\Lambda+1 \leq \rho<1, C>0)$

(I) $-\Lambda+1 \leq \rho<0$

This corresponds to the intersection of curve $y_{1}$ with any value for $y_{2}$ located above the blue horizontal line in Figure 4. Therefore, there are one real positive and two complex eigenvalues. The system is always unstable (no stable equilibrium point) in this region and hence the system is not exponentially convergent. In this parameter range, the state variable $v_{2}^{\prime}$ increases and subsequently $\Pi$ decreases exponentially as a function of $v_{2}^{\prime}$, such that $\rho=1-C \Pi$ reaches zero. The upper limit of the variable $v_{2}^{\prime}$ is determined by solving the equation $\Pi=\frac{K_{0} \Gamma e^{-v_{2}^{\prime}}}{\left(1+K_{0} e^{-v_{2}}\right)^{2}}=\frac{1}{C}$.

(II) $0<\rho<1$

This corresponds to the intersection of curve $y_{1}$ with horizontal line $y_{2}$ located between the red and blue lines in Figure 4. There are one real negative and two complex eigenvalues. The real part of the complex eigenvalues is always negative in this region (Routh-Hurwitz theorem) and the sign does not change in the neighborhood of $\rho=0$ (see Figure 5), therefore no Hopf-bifurcation is obtained in this case. Note that the condition $0<r C_{f b} G \Pi<1$ must be met. Since the plausible values for $C_{f b}$ and $G_{u}$ are much larger than one, the parameter $\Pi$ should take very small values; In other words the sigmoid is saturated. Thus, using self-excitatory feedback leads to saturation 


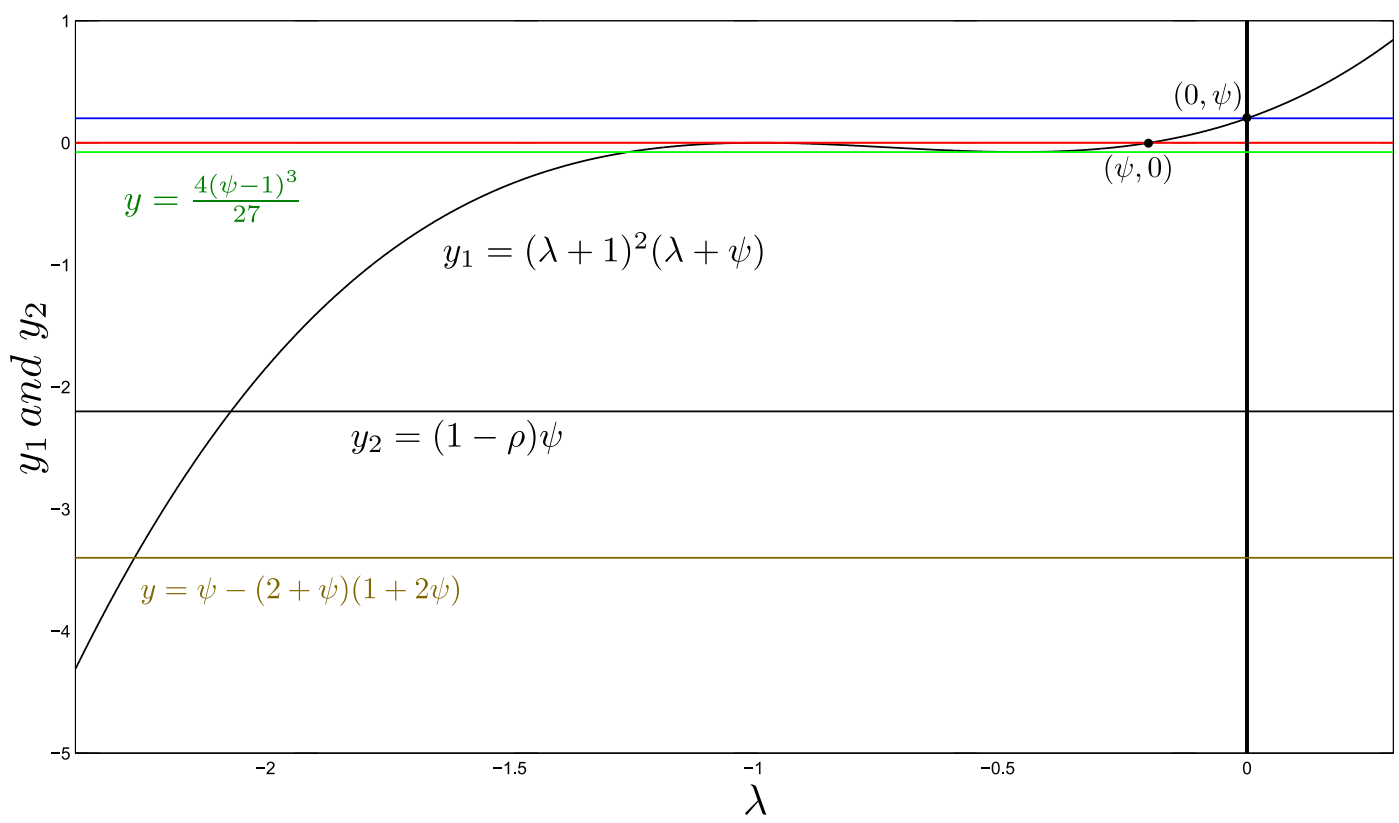

FIGURE 4. Graphical solution of the characteristic equation of the linearized ING circuit near its fixed points. The intersection point of the curve $y_{1}$ and line $y_{2}$ gives the solution. Note that curve $y_{1}$ is always tangent to the $\mathrm{x}$-axis at point $\lambda=-1$. Moreover, $y_{1}$ intersect the $\mathrm{x}$-axis at point $\lambda=-\Psi$. The general shape of curve $y_{1}$ is independent of $\rho$ and within the range of $0<\Psi<1$ this shape is preserved. The red line determines the border between self-excitation (when $\rho<1$ and $y_{1}$ intersects with $y_{2}$ above the line) and self-inhibition (when $\rho>1$ and intersection of $y_{1}$ and $y_{2}$ occurs below the red line). The brown, green and blue borders define the value of $\rho$ at which the type of the eigenvalue of the linearized system changes (see text).

of the population, which is not desired. In conclusion, no oscillatory activity can be obtained in an excitatory neural population with self-excitation.

2.4.2. Inhibitory Self-Feedback $1<\rho \leq \Lambda+1, C<0$

$\left(I^{\prime}\right) 1<\rho<1+\frac{4(1-\psi)^{3}}{27 \psi}$

This corresponds to the intersection of curve $y_{1}$ with horizontal line $y_{2}$ located between red and green lines in Figure 4. Therefore there are three real negative eigenvalues. The linearized system in this case shows overdamped behavior.

$\left(I I^{\prime}\right) 1+\frac{4(1-\psi)^{3}}{27 \psi}<\rho \leq \xi=\frac{(2+\psi)(1+2 \psi)}{\psi}$

This corresponds to the intersection of curve $y_{1}$ with horizontal line $y_{2}$ located between green and brown lines in Figure 4. In this case there are one real negative and two complex eigenvalues. According to the Routh-Hurwitz theorem, the real part of the eigenvalues is negative in this region.

$\left(I I I^{\prime}\right) \xi=\frac{(2+\psi)(1+2 \psi)}{\psi}<\rho \leq \Lambda+1$

This corresponds to the intersection of curve $y_{1}$ with the horizontal line $y_{2}$ located below the brown line in Figure 4. In this case there are one real negative and two complex eigenvalues with positive real parts. The system is not exponentially convergent. According to the Hopfbifurcation theorem, $\rho=\xi$ is the point at which limit cycles emerge, in which the sign of the real eigenvalue remains negative and the signs of the real parts of the complex eigenvalues change from negative to positive.

In Figure 5, the red line demarcates the border between self-excitation (below the line) and selfinhibition (above the line). The border between the green and light blue regions is defined by the line $\rho=1-\frac{4(\psi-1)^{3}}{27 \psi}$ and the border between the dark blue and brown regions is defined by $\rho=\xi=\frac{(2+\psi)(1+2 \psi)}{\psi}$. In the green region, all the eigenvalues are real and negative. In the light blue and dark blue regions 


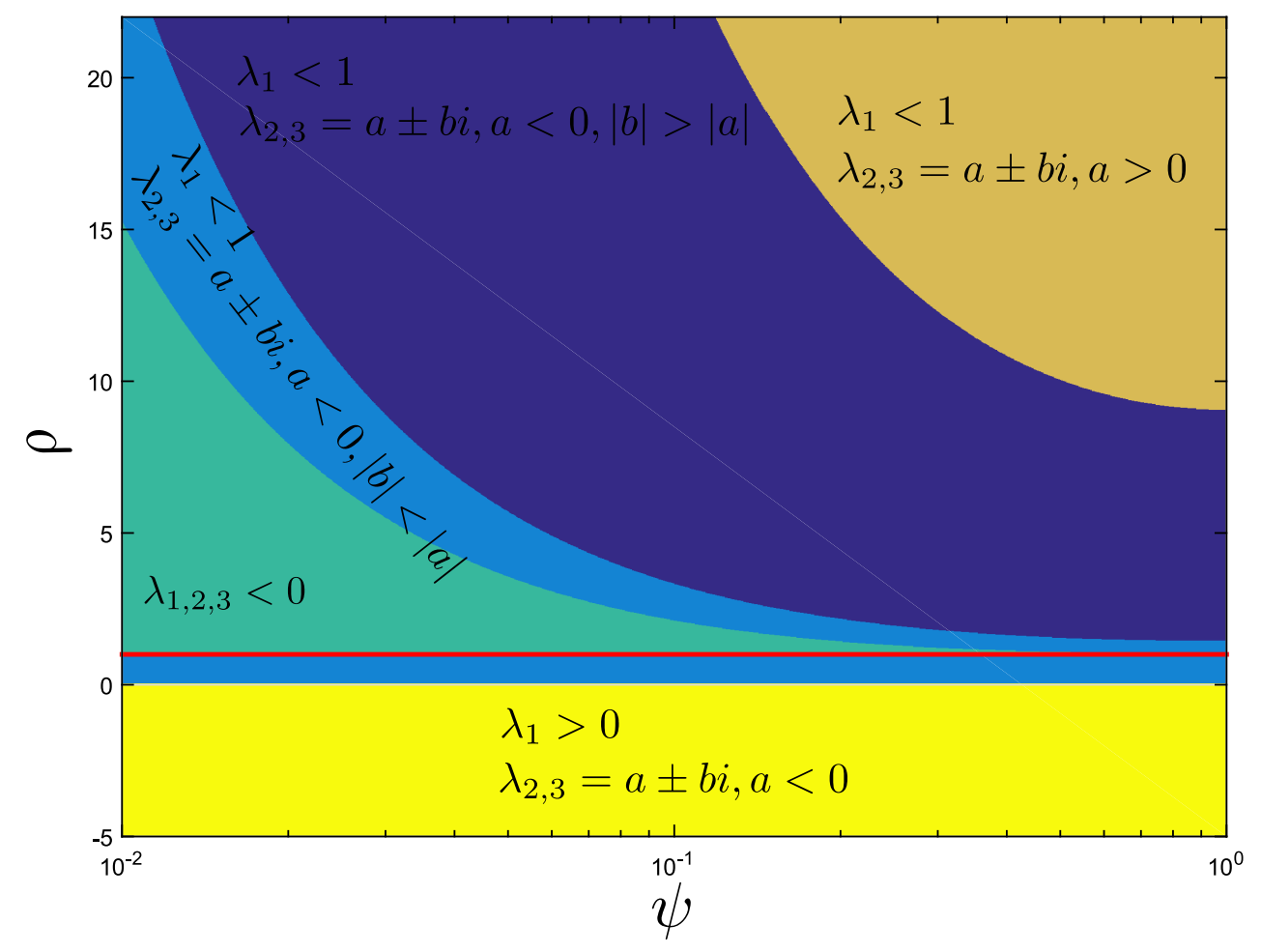

FiguRE 5. Eigenvalues of the linearized dimensionless ING circuit defined in equations 16-18. The solutions of the characteristic equation (Eq. 31) are categorized based on the coefficient parameters $\rho$ and $\Psi$. The red line defines the border between self-inhibition (above the line) and self-excitation (below the line).

a real negative eigenvalue coexists with a complex pair. The real part of the complex eigenvalues is negative in these regions. In the dark blue region, the absolute value of the imaginary part is larger than that of the real part. Therefore, although all the states are stable, the resonance behavior of the system is dominant. Within the dark blue region, forced oscillations can be obtained using a noise source. Periodic fluctuations in noise level induce fluctuations of the same frequency on the resonance frequency of the circuit. In the brown region, the real part of the eigenvalues switches to positive values and a supercritical Hopf-bifurcation occurs. In Ursino, et al. [20], fast activity is generated in a unit with constant self-feedback. Constant self-feedback is equivalent to $\tau \rightarrow 0$ (i.e. $\psi \rightarrow \infty$ ). In this case the sign of the discriminant of the eigenvalues cubic equation when estimated by the sign of $C \Pi$ is always negative. This means that a solution with a real eigenvalue and a complex pair of eigenvalues always exists. The real part of the complex solution when $\tau \rightarrow 0$ (i.e. $\psi \rightarrow \infty$ ) is estimated by $-\frac{\psi}{3}$, which is negative and tends to infinity. Therefore, there is no possibility of a Hopf-bifurcation, since the sign of the real part cannot change by varying any of the system parameters.

\subsection{Selecting the Control Parameters}

Given an inhibitory population with the determined characteristics, a pair of parameters $(\rho, \Psi)$ can be selected and the operating region can be decided. The first parameter $\psi=\frac{1}{\tau_{u} \omega_{u}}$ and $\rho=$ $\left(1-r C_{f b} G_{u} \Pi\left(\frac{\nu_{\max }}{\omega}, e^{r v_{t h}}, r C_{f b} G_{u}, \frac{P_{u}}{\omega_{u}}\right)\right)$ encompasses several of the properties of the inhibitory neural population. We control $\rho$ through the parameter $P_{u}$., i.e. the input level of the inhibitory population. The other parameters (i.e. $r, \nu_{\max }, C_{f b}, G_{u}$ and $\omega_{u}$ ) are specific to the inhibitory neuron population, and therefore are constant or at most vary very slowly in comparison with $P_{u}$. The second parameter, $\Psi=\frac{1}{\tau_{u} \omega_{u}}$ represents the ratio of the synaptic time constant to the self-feedback plasticity time constant. If needed, $\Psi$ can be tuned while leaving $\rho$ unchanged through parameter $\tau_{u}$, as both are independent. The parameter $\tau_{u}$, which represents the plasticity of the self-feedback, is more likely than the ones specific to the neurons to be varying in time. As an example, the relation between $P_{u}$ and $\rho$ is given in Figure 
6 for two values of $\tau_{u}=0.04$ and $\tau_{u}=0.01$, when the remaining parameters are fixed according to Table 1. In Figure 6a, $\tau_{u}$ is set to 0.04 (i.e. $\left.\Psi=0.125\right)$. Therefore the $(\rho, \Psi)$ pair always locates in the resonance (dark blue) region of Figure 6 given any value of the parameter $P_{u}$. However, for $\tau_{u}=0.01$ (i.e. $\Psi=0.5)$, with increment of parameter $P_{u}$ the $(\rho, \Psi)$ pair enters and then exits the limit-cycle (brown region) (Figure 7); Therefore, two Hopf-Bifurcation are expected.
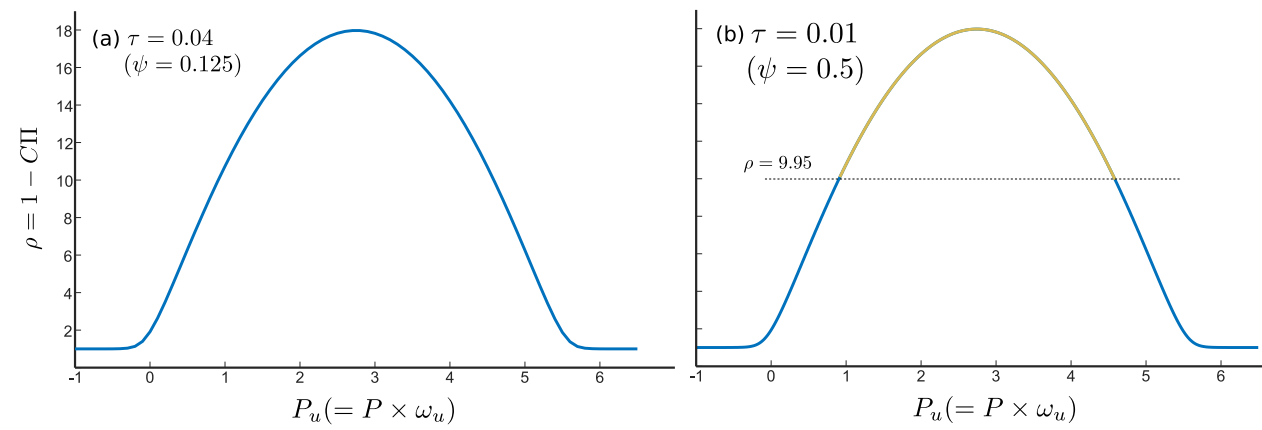

Figure 6. Qualitative Relation between Bifurcation Parameter $\rho$ and $P_{u}$. The remaining parameters of the model are set according to Table 1. (a) For $\tau_{u}=0.04(\Psi=0.125)$ the $(\rho, \Psi)$ pair always locate in the resonance (dark blue) region of Figure 5. (b) For $\tau_{u}=0.01(\Psi=0.5)$ the $(\rho, \Psi)$ pair is in resonance (dark blue) region for $\rho<9.95$ and in limit-cycle (brown) region for $\rho>9.95$. The corresponding ranges for parameter $P_{u}$ and $\rho$ is color coded with blue and brown respectively.

\subsection{Oscillatory Regions}

According to the previous section, the ING circuit can serve as an oscillator in two ways: forced oscillations (dark blue region in Figure 5) or limit cycles (brown region in Figure 5). We will consider in the following sections the methods used in each case to investigate the frequency of oscillations, and the sensitivity of frequency and amplitude to the input $P_{u}$.

\subsubsection{Forced Oscillations}

Forced oscillations can be obtained when the $(\rho, \Psi)$ pair locates in the dark blue region of the parameter space. Since all the state variables of the system are stable, linearization tools can be used to consider the frequency and amplitude of the oscillations. We assume that the input of the sigmoid is $x(t)=n+s(t)$ in which $\mathrm{n}$ is constant and $s(t)$ is a small amplitude (low power) Gaussian white noise. Therefore, the ING circuit can be linearized by replacing the sigmoid block with a variable gain, i.e. the first derivative of the sigmoid function at $u=n$.

$$
\begin{gathered}
\mu=\left.\frac{d \operatorname{Sig}(u)}{d u}\right|_{u=n} \\
\left(\operatorname{Sig}(n)-P_{u}\right) \times \frac{G_{u} C_{f b}}{\omega_{u}}=-n
\end{gathered}
$$

The linearized ING circuit is presented as a block diagram in Figure 7. The transfer function of the linearized ING circuit can be studied using the root locus analysis with the first derivative $\mu$ of the sigmoid function as the gain loop parameter (Figure 8). Note that according to Equation (31), in the linearized system $\rho$ is linearly proportional to $\mu$. Therefore the gain $\mu$ (alike $\rho$ ) can be tuned through parameter $P_{u}$ (Figure 9). Once the parameter $P_{u}$ is chosen to be small enough, we have $n<v_{t h}$ (See Eq. 35) and $\frac{d \mu}{d P_{u}}>0$ ). As a result, changes in parameter $P_{u}$ are proportional to parameter $\mu$ and cause in-phase changes in the resonance frequency (see Figure 8). When $P_{u}$ is large enough, i.e. $n>v_{t h}$ and $\frac{d \mu}{d P_{u}}<0, P_{u}$ changes inversely proportionally to $\mu$ and causes an anti-phase change to the resonance frequency. In Figure 9, the in-phase and anti-phase regions of the ING linearized circuit are presented as a function of parameter $P_{u}$, when the remaining parameters of the model are set according to Table 1 . 


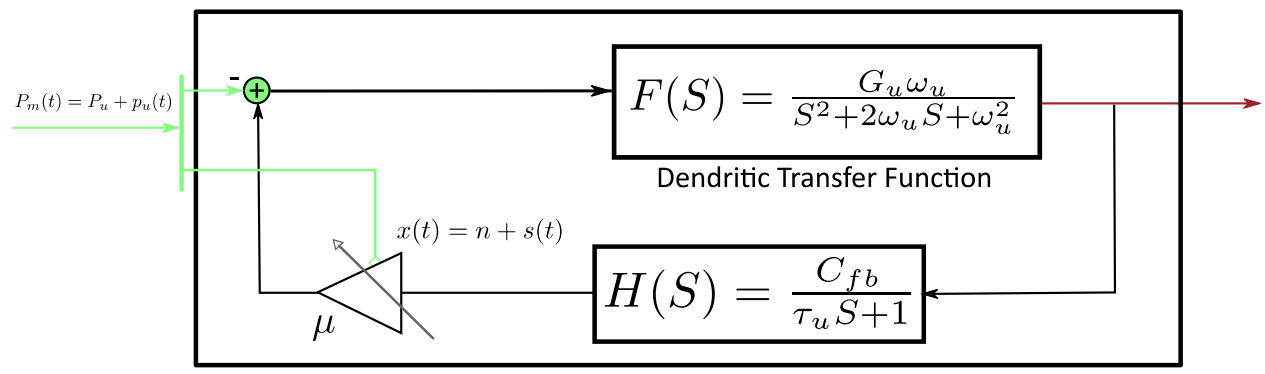

FIGURE 7. Block diagram representation of the linearized ING circuit. When all the state variables of the non-linear system are stable, the nonlinear block can be linearized. In the diagram, the sigmoid function of ING circuit is replaced with its first derivative $\mu$.

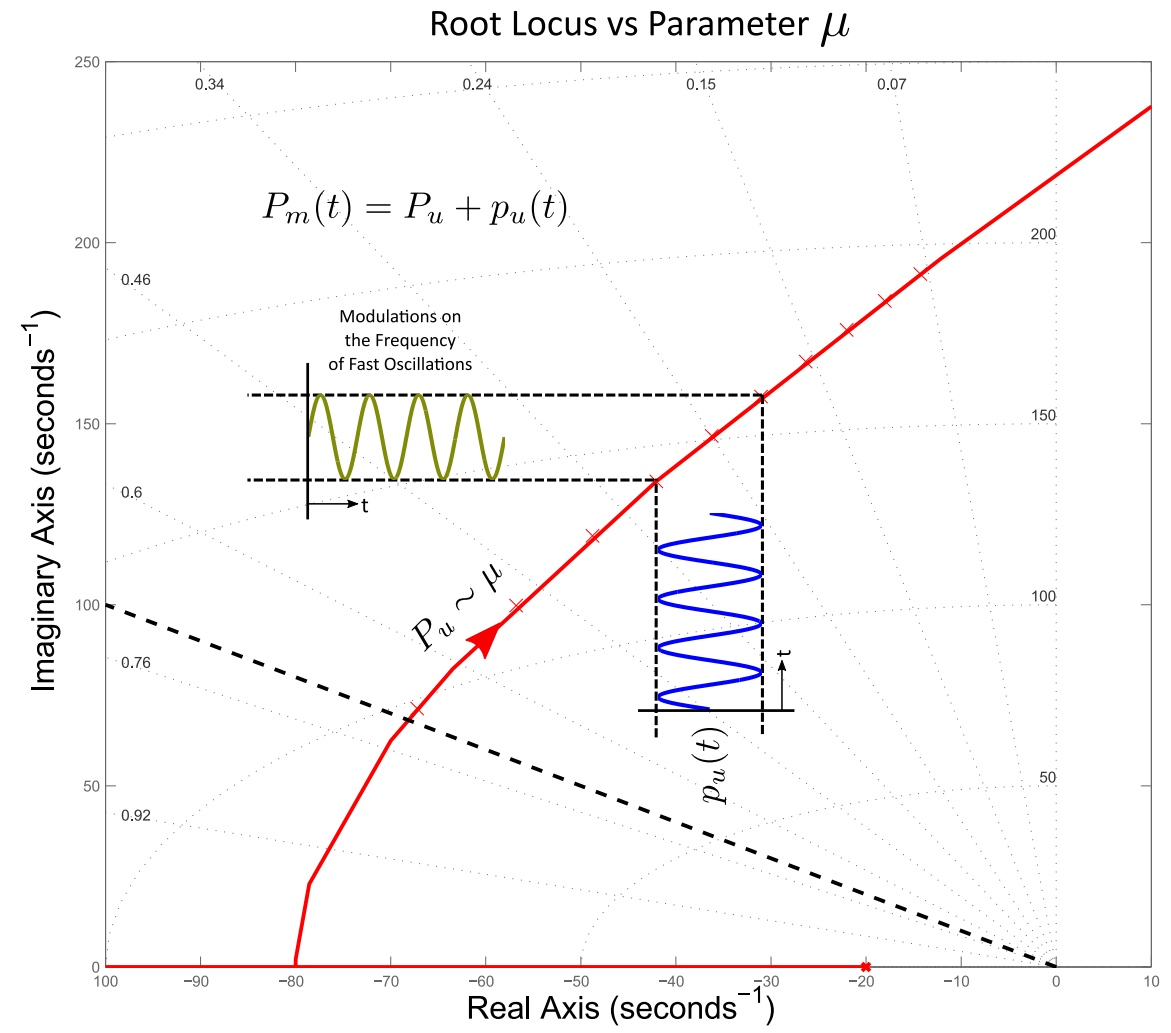

Figure 8. Analysis of frequency modulation using root locus analysis. The resonance frequency (y-axis) increases as the parameter $\mu$ increases. The blue signal indicates changes of parameter $\mu$ in time. The green signal shows the corresponding changes in the location of the resonance frequency.

\subsubsection{Limit Cycles}

In order to investigate the limit cycles, we developed an extended version of the describing function (DF) method. DF is an approximate procedure for analyzing nonlinear systems in which the nonlinear block is estimated with an ideal transfer function with constant phase and amplitude response for all frequencies. This estimation can facilitate the extension of linear techniques in control system theory to nonlinear systems. The phase and amplitude response of the ideal transfer function is calculated at the limit cycle oscillation frequency. The constant phase and amplitude response assumption is not problematic since no other frequency component except the oscillation frequency is expected. The gain for zero frequency is hypothesized to be zero in these approximations when the nonlinear block is odd. Since the sigmoid 


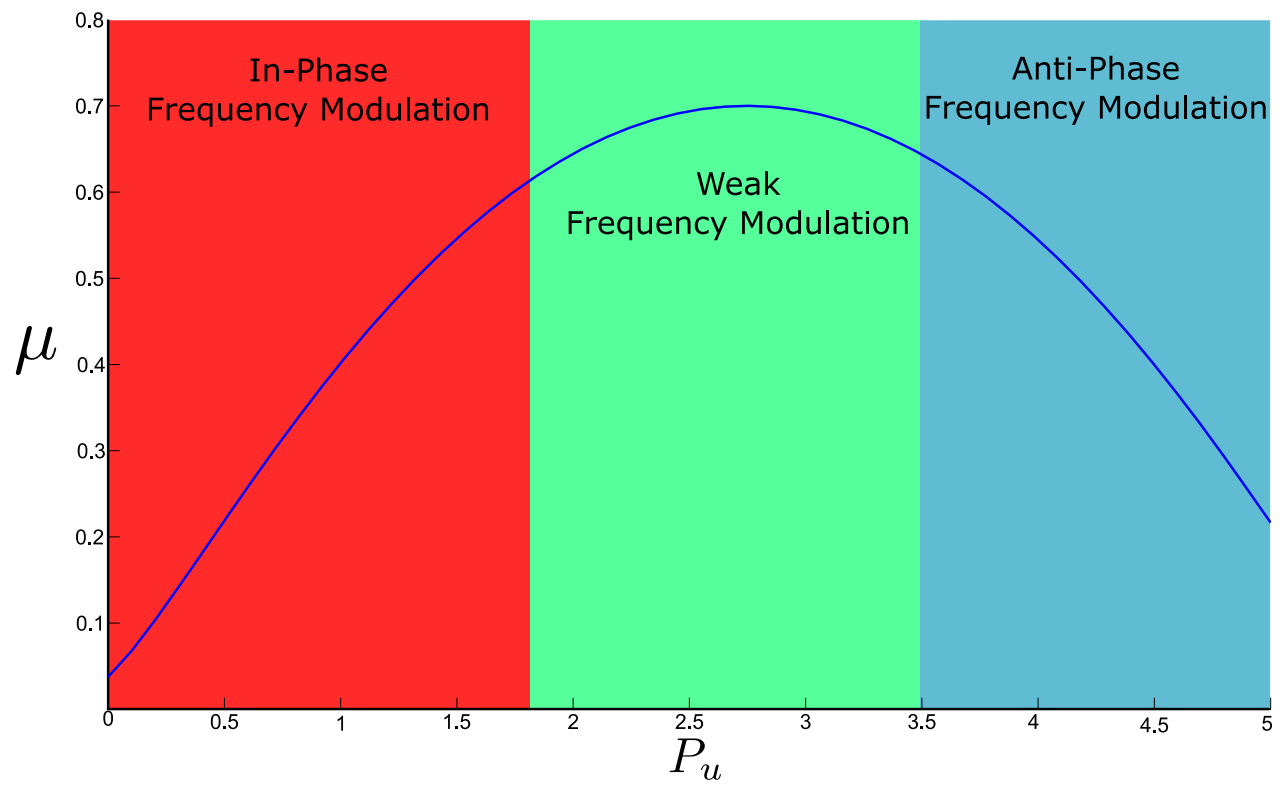

Figure 9. The relation between parameters $P_{u}, \mu$ and the resonance frequency. In the region where $\mu$ is increasing with $P_{u}$, there is an In-Phase change of resonance frequency with parameter $P_{u}$ (red region). When $\mu$ is decreasing with $P_{u}$ the changes in resonance frequency with $P_{u}$ are anti-phase (blue region). In the green region, the change in $\mu$ is small relative to changes in $P_{u}$ and therefore the frequency modulation is weak.

block in the current model is not an odd nonlinear block, an extended version of DF method is needed. In this case the ING circuit is equivalent to the block diagram representation in Figure 10.

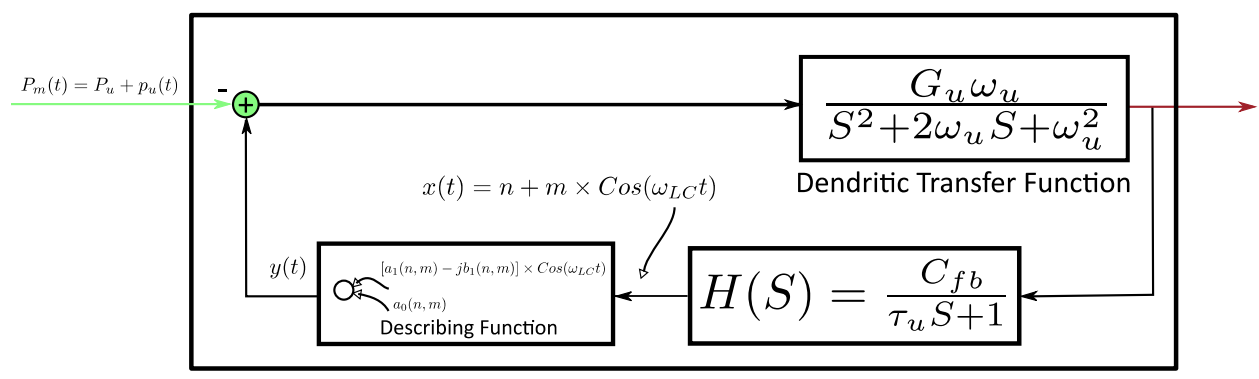

Figure 10. Block Diagram Representation of the ING circuit in Limit-Cycle mode. The sigmoid block is replaced with an ideal transfer function (Describing function) with constant phase and amplitude response for all frequencies.

To start with, we assume that an oscillatory activity with constant amplitude $\mathrm{m}$ and time-invariant part $\mathrm{n}$ exists right before the sigmoid block. This signal can be described as follows:

$$
x(t)=n+m \times \cos \left(\omega_{L C} t\right)
$$

Therefore the output of the sigmoid block can be obtained using the Fourier series:

$$
y(t)=\operatorname{Sig}(x(t))=a_{0}+\sum_{k=1}^{\infty} a_{k} \cos \left(k \omega_{L C} t\right)+\sum_{l=1}^{\infty} b_{l} \sin \left(l \omega_{L C} t\right)
$$

One necessary condition for steady oscillation is that the constant value at the output of DF, $a_{0}$, returns back to its initial value while propagating through the loop. This means the following equation must be satisfied: 


$$
a_{0}(m, n)=\frac{n \omega_{u}}{G_{u} C_{f b}}-P_{u}
$$

and $a_{0}(m, n)$ is the constant value calculated using the following equation:

$$
a_{0}(m, n)=\frac{\omega_{L C}}{2 \pi} \int_{0}^{\frac{2 \pi}{\omega_{L C}}} \operatorname{Sig}(n+m \times \cos (t)) d t
$$

Equation (2.38) has no solution for $P_{u}<0$; for $P_{u}>0$ the solution is a line in the $m-n$ plane. The amplitude of the second and higher order harmonics (Equation 2.37) are all negligible and are filtered out by the dendritic transfer function, which is essentially a low-pass filter. Therefore only the coefficients of the first harmonic are important and can be obtained using:

$$
\begin{aligned}
& a_{1}(m, n)=\frac{\omega_{L C}}{\pi} \int_{0}^{\frac{2 \pi}{\omega_{L C}}} \operatorname{Sig}(n+m \times \cos (t)) \cos (t) d t \\
& b_{1}(m, n)=\frac{\omega_{L C}}{\pi} \int_{0}^{\frac{2 \pi}{\omega_{L C}}} \operatorname{Sig}(n+m \times \cos (t)) \sin (t) d t
\end{aligned}
$$

If the necessary condition (Equation 2.38) is met, a sufficient condition for emergence of limit cycles is that the describing function intersects with the loop transfer function. This condition is known as the describing function equation, as follows:

$$
1+N(n, m) T(j \omega)=0
$$

Where $T(j \omega)$ is the transfer function of the linear part of the closed loop:

$$
T(j \omega)=F(j \omega) \times H(j \omega)=\frac{G_{u} \omega_{u}}{-\omega^{2}+2 \omega_{u} \omega j+\omega_{u}^{2}} \times \frac{C_{f b}}{1+\tau_{u} \omega j}
$$

and $N(n, m)$ is the describing function of the nonlinear block as a function of time-invariant part (n) and amplitude $(\mathrm{m})$ of the input oscillation. It can be described using:

$$
N(n, m)=\frac{a_{1}-j b_{1}}{m}
$$

$b_{1}=0$ due to the symmetry of the sigmoid. Therefore, $N(n, m)$ is always real. Hence, the describing function equation (Eq. 44) yields the following two equations:

$$
\begin{gathered}
\angle T\left(j \omega_{L C}\right)=\tan ^{-1}\left(\frac{\omega_{L C}\left(2 \omega_{u}+\tau_{u}\left(\omega_{u}^{2}-\omega_{L C}^{2}\right)\right)}{\omega_{L C}^{2}\left(1+2 \tau_{u} \omega_{u}\right)-\omega_{u}^{2}}\right)=0 \\
\left|a_{1}\right|=\frac{m}{\left|T\left(j \omega_{L C}\right)\right|}
\end{gathered}
$$

From Equation (2.45) we get:

$$
f_{L C}=\frac{\omega_{L C}}{2 \pi}=\frac{1}{2 \pi} \sqrt{\frac{2 \omega_{u}}{\tau_{u}}+\omega_{u}^{2}}
$$

and Equation (2.46) can be rewritten as:

$$
m=\frac{\left|a_{1}\right| \tau_{u} G_{u} C_{f b}}{2\left(\tau_{u} \omega_{u}+1\right)^{2}}
$$

Equations 2.38 and 2.48 always have a solution in the brown region of Figure 5 that yields a constant value and amplitude of the limit cycle oscillations. A graphical solution for the describing function 
equation is presented in Figure 11. In this figure the model parameters are set according to Table 1 and the parameter $P_{u}=1$. The frequency of the oscillations can be re-written as a function of $\Psi=\frac{1}{\omega_{u} \tau_{u}}$ ):

$$
f_{L C}=\frac{\omega_{u}}{2 \pi} \sqrt{2 \psi+1}
$$

Since $0<\Psi<1$, the frequency of the limit cycle can be chosen between $\frac{\omega_{u}}{2 \pi}$ and $\frac{\sqrt{3} \omega_{u}}{2 \pi}$. Moreover, we have $\frac{\partial f_{L C}}{\partial P_{u}}=0$, which means that the frequency is not a function of input $P_{u}$.

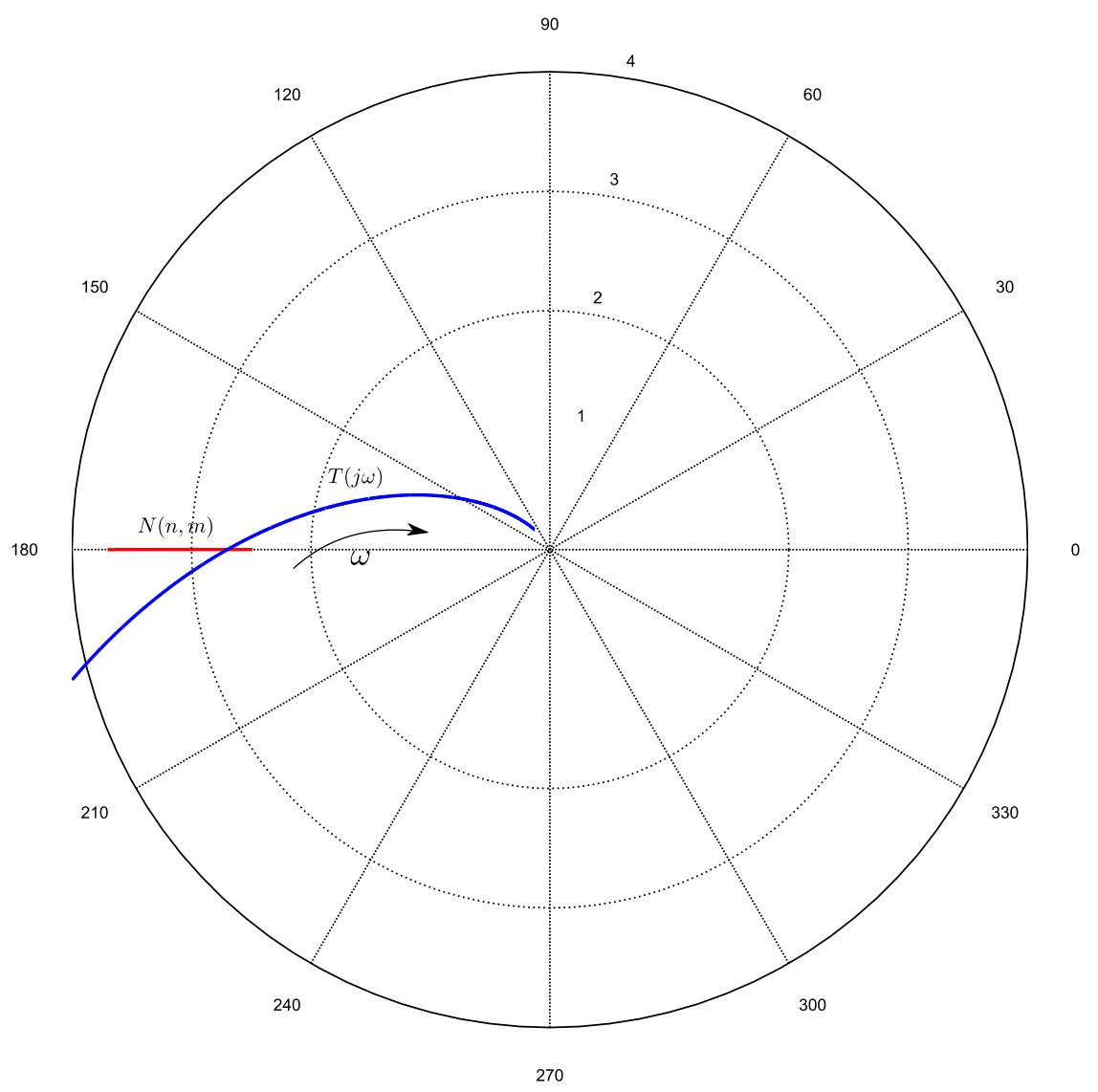

FiguRE 11. Graphical solution of the describing function equation. The intersection of the describing function (red line) with the loop transfer function (blue line) gives the frequency and amplitude of the oscillations.

We performed a bifurcation analysis using the AUTO software [36], for the ING circuit described in Equations (2.7-2.9), with $P_{u}$ as the bifurcation parameter. The bifurcation diagram is presented in Figure 12. A Hopf bifurcation occurs two times with increasing parameter $P_{u}$; the first causes the emergence of the limit cycle and after the second bifurcation the limit cycle dies out. Right after the first bifurcation, with larger values of $P_{u}$, the amplitude of the limit cycles increases (observe $m_{1}$ and $m_{2}$ in Figure 12). Correspondingly, the amplitude of the oscillation is in-phase with fluctuations in $P_{u}$. Before the second bifurcation the amplitude of the oscillations gets smaller, the larger the value of $P_{u}$. Correspondingly, the amplitude of limit cycle changes in anti-phase with fluctuations in $P_{u}$. The in-phase and anti-phase amplitude modulation regions of the ING circuit as a function of parameter $P_{u}$ are graphically represented in Figure 13.

\subsection{Simulation of Frequency and Amplitude Modulations in the ING circuit}

Here, in the main input of the ING circuit $P_{m}(t)=P_{u}+p_{u}(t)$, we define $p_{u}(t)=\operatorname{mSin}\left(2 \pi f_{m} t\right)$ which is an ideal deterministic sine wave $\left(f_{m}=4 \mathrm{~Hz}\right)$ which represents Delta-Theta oscillation in brain signals. 


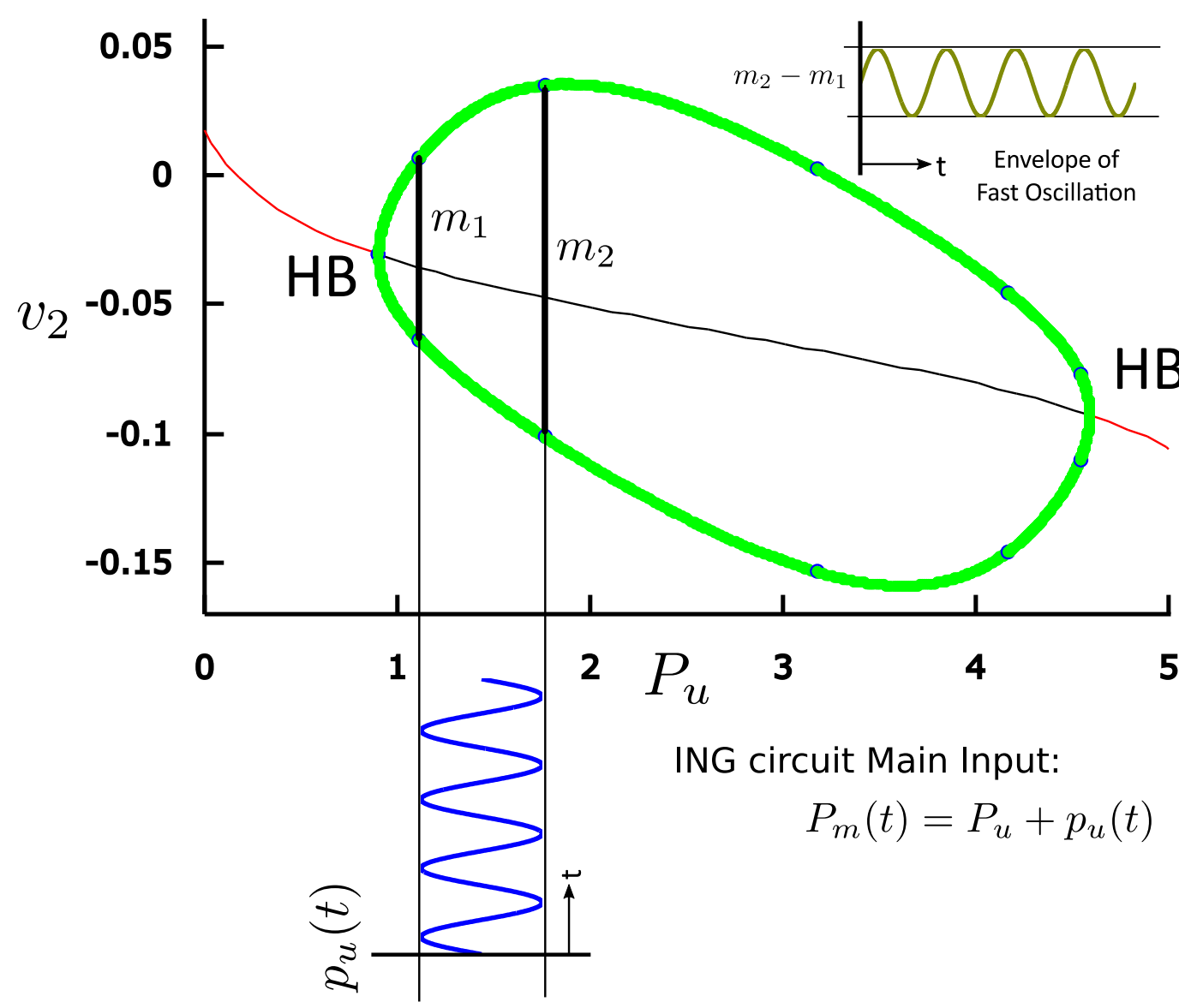

FiguRE 12. Bifurcation diagram of the ING circuit (Eq 7-9) with $P_{u}$ as the bifurcation parameter. The blue signal indicates oscillations of $p_{u}(t)$ in time $t$. The green signal shows the corresponding changes in the amplitude of the oscillations. $m_{1}$ and $m_{2}$ are peak-to-peak amplitude of the limit-cycle oscillations and HB stands for HopfBifurcation.

Moreover, $P_{u}$ is replaced with $N\left(P_{u}, \sigma^{2}\right)$, which is a stochastic term (Gaussian white noise) and represents un-modeled activity affecting the ING circuit. $P_{u}$ determines the mean excitation level and $\sigma$ indicates the uncertainty around this value. Frequency and amplitude modulations are simulated for both in-phase and anti-Phase using the ING circuit presented in Figure 3b (Equations 2.7-2.9). This results in four simulations. Table 1 gives the fixed parameter values for all simulation runs. For frequency modulation we chose $\Psi=0.125$ (i.e. $\tau_{u}=0.04$ ) which assures the pair $(\Psi, \rho)$ locates in resonance (dark blue) region of Figure 5, given any plausible value of $\rho$. For $\mu=0.4$, which is about half of its maximum value and corresponds to $\rho=10.7$, we get $P_{u}$ values for in-phase and anti-phase regions 1 and 4.5 respectively (Figure 9). For amplitude modulation we set $\Psi=0.5$ (i.e. $\tau_{u}=0.01$ ) and $\rho=11$ which locates the $(\Psi, \rho)$ pair in the limit-cycle (brown) region of Figure 5. The corresponding in-phase and anti-phase values for $P_{u}$ are calculated as 1 and 4.5 respectively. In Table 2, the parameters that vary between simulations are summarized. Note that to switch between in-phase and anti-phase modes, only the parameter $P_{u}$ is changed.

TABLE 1. The parameters of the ING circuit common in all the simulations

\begin{tabular}{|c|c|c|c|c|c|c|}
\hline$C_{f b}$ & $\omega_{u}^{-1}$ & $G_{u}$ & $v_{t h}$ & $\nu_{\max }$ & $r$ & $\sigma$ \\
\hline 97 & $200^{-1}$ & 50 & 6 & 5 & 0.56 & 0.07 \\
\hline
\end{tabular}




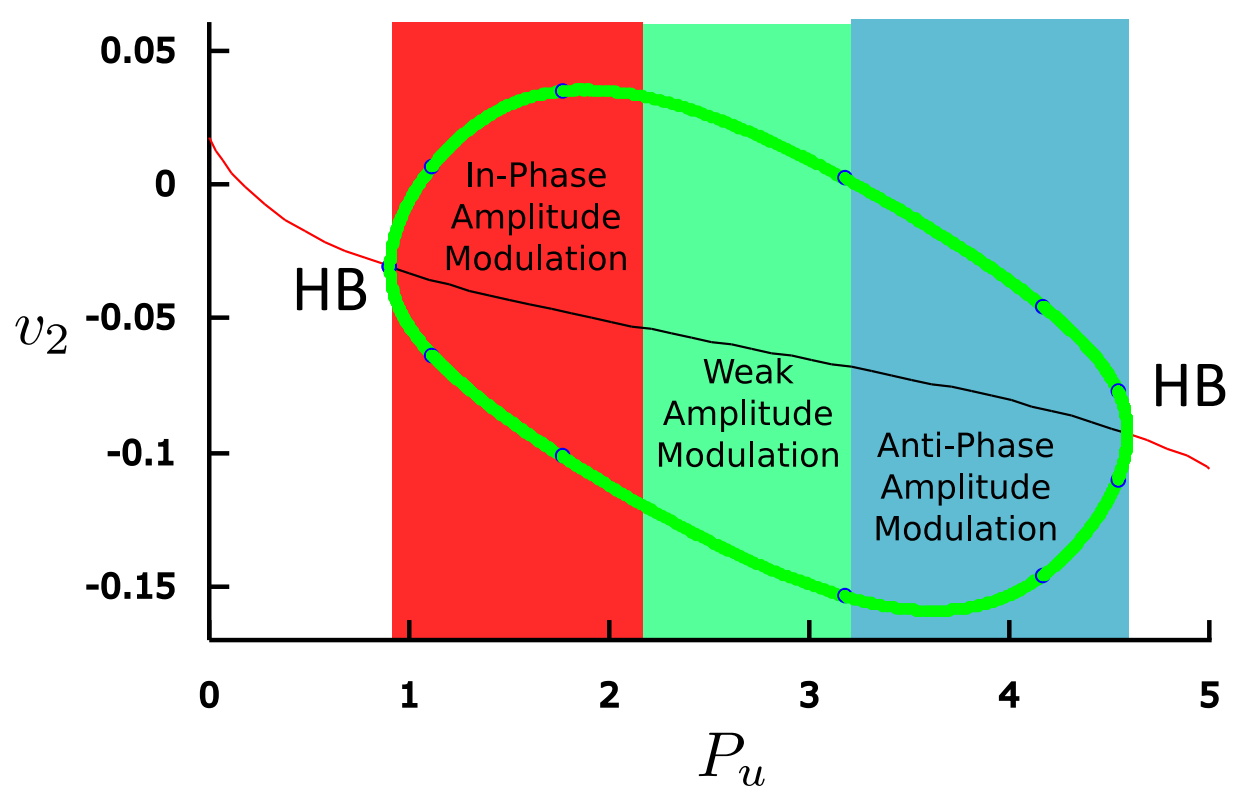

FiguRE 13. The relation between parameters $P_{u}$ and the amplitude modulations. In the region where $P_{u}$ is close to the first Hopf-Bifurcation, In-Phase amplitude of the limit-cycle varies with parameter $P_{u}$ (red region). When $P_{u}$ is close to the second HopBifurcation, anti-phase amplitude of the limit-cycle oscillations varies with parameter $P_{u}$ (blue region). In the green region, amplitude of the limit cycle is relatively insensitive to changes in $p_{u}(t)$ and therefore the amplitude modulation is weak. The bifurcation diagram is obtained using the values in Table 1 and for $\tau_{u}=0.01 \mathrm{~s}$.

TABLE 2. Parameter values for simulating different types of modulations

\begin{tabular}{|c|c|c|c|c|c|c|}
\hline Type of Modulation & $P$ & $M$ & $P_{u}$ & $\Psi=\frac{1}{\omega_{u} \tau_{u}}$ & $\tau_{u}$ & $m$ \\
\hline Frequency Modulation (In-Phase) & 10.7 & 0.4 & 1 & 0.125 & 0.04 & 1.5 \\
\hline Frequency Modulation (Antn-Phase) & 10.7 & 0.4 & 4.5 & 0.125 & 0.04 & 1.5 \\
\hline Amplitude Modulation (In-Phase) & 11 & - & 1 & 0.5 & 0.01 & 0.5 \\
\hline Amplitude Modulation (Anti-Phase) & 11 & - & 4.5 & 0.5 & 0.01 & 0.5 \\
\hline
\end{tabular}

\subsection{Simulation Specifications}

The model was implemented and ran in Matlab/Simulink 8.0 (R2012b), using a $4^{\text {th }}$ order Runge-Kutta method with fixed time step of 0.001 seconds. The number of iterations was chosen sufficiently large to assure stationarity of the dynamics. Transient dynamics were omitted from data analyses and graphical presentations of the results. For visualization the ideal sinewave is omitted from the output of the model using a notch filter centered at $4 \mathrm{~Hz}$.

\section{Results}

\subsection{ING Circuit in Frequency Modulation Mode}

Figure 14 shows the output of the ING circuit in frequency modulation mode according to the parameter values in Table 1 and Table 2 (first and second rows). For $P_{u}=1$ (Figure 14a) an in-phase frequency modulation is obtained. For every positive phase of the Theta band input signal (Figure 14c), the frequency of fast oscillations was increased and for every negative phase it was decreased. Anti-phase frequency modulation is observed for $P_{u}=4.2$ (Figure 14b). The fast oscillation was decreased and increased in frequency within, respectively, the positive and negative phases of the Theta input signal. 
(a)

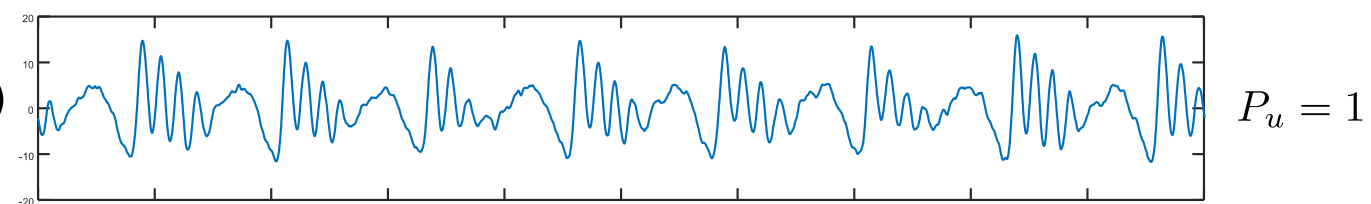

(b)

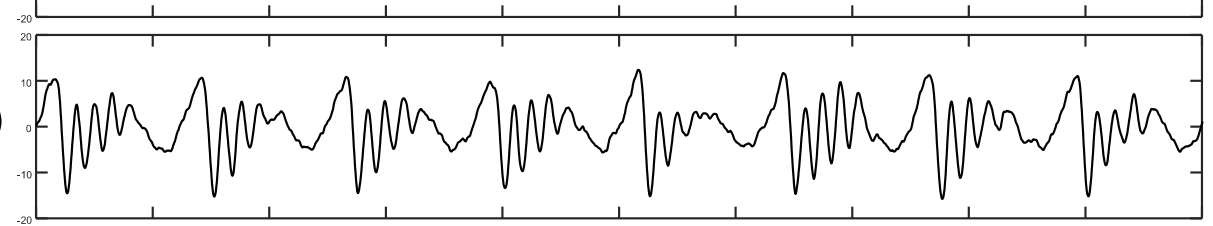

$P_{u}=4.4$

(C)

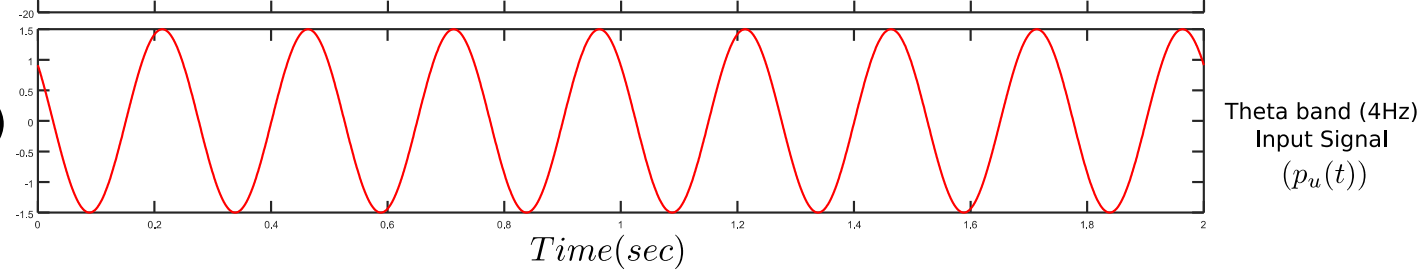

FIGURE 14. Simulation of frequency modulation in the ING circuit. (a) In-phase frequency modulation (b) Anti-phase frequency modulation (c) Theta band (4Hz) input signal.

\subsection{ING Circuit in Amplitude Modulation Mode}

Figure 15 demonstrates the performance of the ING circuit in amplitude modulation mode, using the values of Table 1 and Table 2 (third and fourth rows). For $P_{u}=1$ an in-phase amplitude modulation occurred (Figure 15a). The instantaneous amplitude of the fast oscillation is increased and decreased for every positive and negative phases of Theta band input signal (Figure 15c) respectively. Figure 15b demonstrates anti-phase amplitude modulation for $P_{u}=4.4$ in which the instantaneous amplitude of the fast oscillation is negatively correlated with Theta band input signal.

(a)

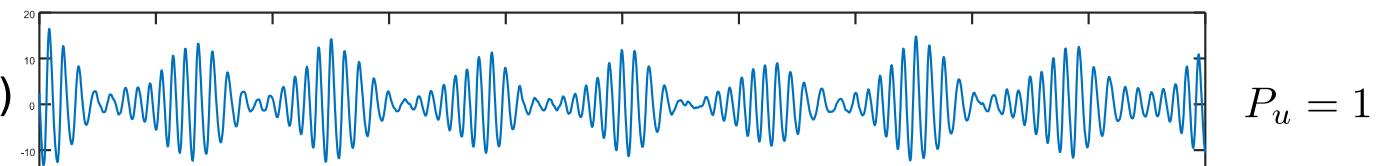

(b)

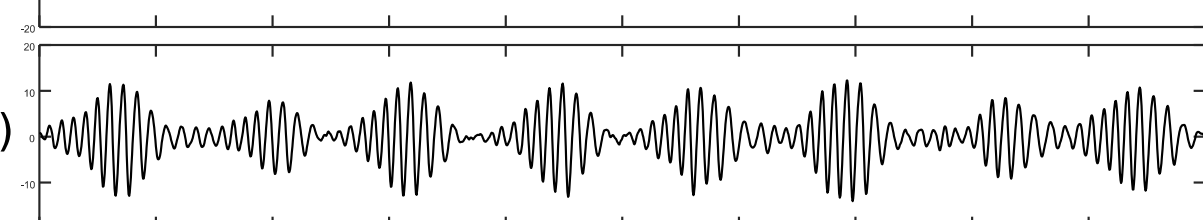

$P_{u}=4.4$

(c)

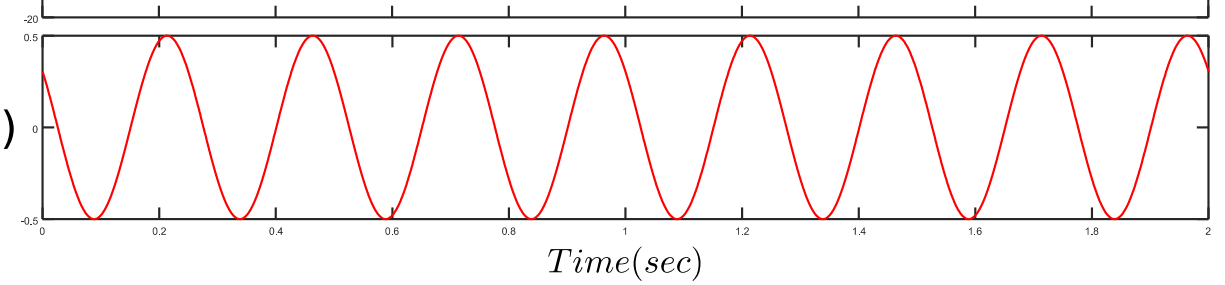

Theta band $(4 \mathrm{~Hz})$ Input Signal

$\left(p_{u}(t)\right)$

FIGURE 15. Simulation of amplitude modulation in the ING circuit. (a) In-phase amplitude modulation (b) Anti-phase amplitude modulation (c) Theta band (4Hz) input signal. 


\section{Discussion}

We studied analytically an Interneuron Gamma (ING) circuit designed for the purpose of obtaining CFC between slow and fast oscillations in a Neural Mass Model. The ING circuit was utilized in Chehelcheraghi [7] for modeling those forms of CFC: PFC, PAC, FFC, AAC, and AFC [23] that involve amplitude and/or frequency modulation on the fast oscillations. We determined analytically for the ING circuit the parameter region for which the circuit can switch between amplitude and frequency modulation modes. We showed that the behavior of the ING circuit can be described using only two parameters. The first parameter is $\rho$, which is a function of the mean excitation level input $P_{u}$. The parameter $\rho$ encompasses almost all the intrinsic properties of the inhibitory neural population. The second parameter, $\Psi$ represents the ratio of the synaptic time constant to the self-feedback plasticity time constant. Higher values of $\Psi$ indicate faster synaptic plasticity. As the dynamic of the synaptic plasticity cannot be faster the the synapse dynamics itself, $\Psi$ varies between 0 and 1 . The analysis predicts that for larger values of $\Psi$, the probability increases that limit cycle oscillations emerge in brain activity. This is in accordance with computational [9] and experimental studies [2][32], in which synaptic depression is emphasized as a key factor in the emergence of fast oscillations. To switch between amplitude and frequency modulation mode, the ING circuit structure and those parameters which desribe the intrinsic properties of the neurons, e.g strength and time constant of the synaptic transfer function did not have to be changed. Rather, the level of excitation of the ING circuit (parameter $P_{u}$ ) was crucial. Thus, the simulation results predict that, as the mean input level to a cortical region changes, the mode of the ING oscillation will change to a different mode. Moreover, within a specific type of modulation, the in-phase or anti-phase properties of the modulations depend on the mean excitation level.

Acknowledgements. This research was supported by an Odysseus grant from the Flemish organizations for science (FWO) awarded to Cees van Leeuwen

\section{References}

[1] M. Antonakakis et al., Altered cross-frequency coupling in resting-state MEG after mild traumatic brain injury. International Journal of Psychophysiology 102 (2016), 1-11 [doi:10.1016/j.ijpsycho.2016.02.002].

[2] M. Bartos, I. Vida, and P. Jonas, Synaptic mechanisms of synchronized gamma oscillations in inhibitory interneuron networks. Nature Reviews Neuroscience 8 (2007), No. 1, 45-56 [doi:10.1038/nrn2044].

[3] M. Bekisz and A. Wrobel, Coupling of beta and gamma activity in corticothalamic system of cats attending to visual stimuli. NeuroReport 10 (1999), No. 17, 3589-3594.

[4] C. Borgers and N. Kopell, Synchronization in Networks of Excitatory and Inhibitory Neurons with Sparse, Random Connectivity. Neural Computation 15 (2003), No. 3, 509-538 [doi:10.1162/089976603321192059].

[5] G. Buzsaki and X. J. Wang, Mechanisms of Gamma Oscillations. Annu. Rev. Neurosci. 35 (2012), No. 1, 203-225 [doi:10.1146/annurev-neuro-062111-150444].

[6] R. T. Canolty and R. T. Knight, The functional role of cross-frequency coupling. Trends in Cognitive Sciences 14 (2010), No. 11, 506-515 [doi:10.1016/j.tics.2010.09.001].

[7] M. Chehelcheraghi, C. van Leeuwen, E.Steur, C. Nakatani A neural mass model of cross frequency coupling. PLOS ONE 12 (2017), No. 4, e0173776 [doi: 10.1371/journal.pone.0173776].

[8] M. Chehelcheraghi et al., A neural mass model of phase-amplitude coupling. Biological Cybernetics 110 (2016), No. 2-3, 171-192 [doi:10.1007/s00422-016-0687-5].

[9] W. M. Connelly, Autaptic Connections and Synaptic Depression Constrain and Promote Gamma Oscillations. PLOS ONE 9 (2014), No. 2, e89995 [doi:10.1371/journal.pone.0089995].

[10] J. Csicsvari et al., Mechanisms of Gamma Oscillations in the Hippocampus of the Behaving Rat. Neuron 37 (2003), No. 2, 311-322 [doi:10.1016/S0896-6273(02)01169-8].

[11] O. David and K. J. Friston, A neural mass model for MEG/EEG:: coupling and neuronal dynamics. NeuroImage 20 (2003), No. 3, 1743-1755 [doi:10.1016/j.neuroimage.2003.07.015].

[12] G. Deco et al., The Dynamic Brain: From Spiking Neurons to Neural Masses and Cortical Fields. PLOS Computational Biology 4 (2008), No. 8, e1000092 [doi:10.1371/journal.pcbi.1000092].

[13] E. J. Doedel et al., AUTO-07P: Continuation and bifurcation software for ordinary differential equations. (2007).

[14] I. C. Fiebelkorn et al., Cortical cross-frequency coupling predicts perceptual outcomes. NeuroImage 69 (2013), 126-137 [doi:10.1016/j.neuroimage.2012.11.021].

[15] F. Grimbert and O. Faugeras, Bifurcation analysis of Jansen's neural mass model. Neural Computation 18 (2006), No. 12, 3052-3068 [doi:10.1162/neco.2006.18.12.3052].

[16] E. Guatteo et al., Temperature Sensitivity of Dopaminergic Neurons of the Substantia Nigra Pars Compacta: Involvement of Transient Receptor Potential Channels. Journal of Neurophysiology 94 (2005), No. 5, 3069-3080 [doi:10.1152/jn.00066.2005].

[17] N. Hajos and O. Paulsen, Network mechanisms of gamma oscillations in the CA3 region of the hippocampus. Neural Networks 22 (2009), No. 8, 1113-1119 [doi:10.1016/j.neunet.2009.07.024]. 
[18] J. A. Hamalainen et al., Reduced phase locking to slow amplitude modulation in adults with dyslexia: An MEG study. NeuroImage 59 (2012), No. 3, 2952-2961 [doi:10.1016/j.neuroimage.2011.09.075].

[19] F. Hucho and C. Weise, Ligand-Gated Ion Channels. Angewandte Chemie International Edition 40 (2001), No. 17, 3100-3116 [doi:10.1002/1521-3773(20010903)40:17;3100::AID-ANIE3100¿3.0.CO;2-A].

[20] A. Hyafil et al., Neural Cross-Frequency Coupling: Connecting Architectures, Mechanisms, and Functions. Trends in Neurosciences 38 (2015), No. 11, 725-740 [doi:10.1016/j.tins.2015.09.001].

[21] J. Jackson, R. Goutagny, and S. Williams, Fast and Slow Gamma Rhythms Are Intrinsically and Independently Generated in the Subiculum. Journal of Neuroscience 31 (2011), No, 34, 12104-12117 [doi:10.1523/JNEUROSCI.137011.2011].

[22] B. H. Jansen and V. G. Rit, Electroencephalogram and visual evoked potential generation in a mathematical model of coupled cortical columns. Biological Cybernetics. 73 (1995), No. 4, 357-366 [doi:10.1007/BF00199471].

[23] O. Jensen and L. L. Colgin, Cross-frequency coupling between neuronal oscillations. Trends in Cognitive Sciences 11 (2007), No. 7, 267-269 [doi:10.1016/j.tics.2007.05.003].

[24] V. Jirsa and V. MÃ $\frac{1}{4} l$ ler, Cross-frequency coupling in real and virtual brain networks. Frontiers in Computational Neuroscience 7 (2013) [doi:10.3389/fncom.2013.00078].

[25] L. S. Leung, Nonlinear feedback model of neuronal populations in hippocampal CAl region. Journal of Neurophysiology 47 (1982), No. 5, 845-868.

[26] R. M. McKernan and P. J. Whiting, Which GABAA-receptor subtypes really occur in the brain. Trends in Neurosciences 19(1996), No. 4, 139-143 [doi:10.1016/S0166-2236(96)80023-3].

[27] M. Siegel, T. H. Donner, and A. K. Engel, Spectral fingerprints of large-scale neuronal interactions. Nature Reviews Neuroscience 13 (2012), No. 2, 121-134 [doi:10.1038/nrn3137].

[28] F. H. L. da Silva et al., Model of brain rhythmic activity, Kybernetik 15 (1974), No. 1, 27-37 [doi:10.1007/BF00270757]

[29] A. Spiegler et al., Bifurcation analysis of neural mass models: Impact of extrinsic inputs and dendritic time constants. Neuroimage 52 (2010), No. 3, 1041-1058.

[30] A. B. L. Tort et al., Dynamic cross-frequency couplings of local field potential oscillations in rat striatum and hippocampus during performance of a T-maze task. Proceedings of the National Academy of Sciences 105 (2008), No. 51, 20517-20522 [doi:10.1073/pnas.0810524105].

[31] J. Touboul and O. Faugeras, Codimension Two Bifurcations and Rythms in Neural Mass Models. ArXiv Prepr. ArXiv09072718 (2009).

[32] R. D. Traub et al., Transient Depression of Excitatory Synapses on Interneurons Contributes to Epileptiform Bursts During Gamma Oscillations in the Mouse Hippocampal Slice. Journal of Neurophysiology 94 (2005), No. 2, 1225-1235 [doi:10.1152/jn.00069.2005].

[33] M. Ursino, F. Cona, and M. Zavaglia, The generation of rhythms within a cortical region: Analysis of a neural mass model. NeuroImage 52 (2010), No. 3, 1080-1094 [doi:10.1016/j.neuroimage.2009.12.084].

[34] J. Vriens, B. Nilius, and T. Voets, Peripheral thermosensation in mammals. Nature Reviews Neuroscience 15 (2014), No. 9, 573-589 [doi:10.1038/nrn3784].

[35] X.-J. Wang and G. Buzsaki, Gamma Oscillation by Synaptic Inhibition in a Hippocampal Interneuronal Network Model. Journal of Neuroscience 16 (1996), No. 20, 6402-6413.

[36] F. Wendling et al., Epileptic fast activity can be explained by a model of impaired GABAergic dendritic inhibition European Journal of Neuroscience 15 (2002), No. 9, 1499-1508.

[37] M. A. Whittington et al., Multiple origins of the cortical gamma rhythm. Developmental Neurobiology 71 (2011), No. 1, 92-106 [doi:10.1002/dneu.20814].

[38] M. Zavaglia et al., A neural mass model for the simulation of cortical activity estimated from high resolution EEG during cognitive or motor tasks Journal of Neuroscience Methods 157 (2006), No. 2, 317-329 [doi:10.1016/j.jneumeth.2006.04.022]. 